\title{
The Generalized of $\cosh (\xi)$ Expansion Method And ItsApplication To Derivative Schrödinger Equation
}

\author{
Alaaeddin Amin Moussa, Lama Abdulaziz Alhakim \\ MIS department, College of Business \& Economics/Qassim University, Buraidah, K.S.A
}

\begin{abstract}
In this paper, an efficient generalized of $\cosh (\xi)$ expansion method is proposed to seek traveling wave solutions of the derivative Schrödinger equation. The traveling wave solutions are expressed in terms of the hyperbolic and trigonometric functions. It is shown that the method is straightforward and effective for solving nonlinear evolution equations in mathematical physics.
\end{abstract}

Keywords: Generalized of $\cosh (\xi)$ Expansion Method; Exact Solutions; Derivative Schrödinger Equation.

\section{Introduction}

Nonlinear evolution equations (NLEEs) are widely used as models to describe many important complex physical phenomena in various fields of science, such as plasma physics, nonlinear optics, solid state physics, chemical kinematics, fluid mechanics, chemistry, biology and so on. Thus, establishing exact traveling wave solutions of NLEEs is very important to better understand nonlinear phenomenas as well as other real-life applications.

In the recent years, a wide range of methods have been developed to generate analytical solutions of nonlinear partial differential equations. Among these methods are the $\left(\frac{G^{\prime}}{G}\right)$ expansion method $[1,2]$, the $\left(\frac{G^{\prime}}{G}, \frac{1}{G}\right)$ expansion method [3], the $\exp (-\phi(\xi))$ expansion method [4,5], the generalized of $\exp (-\phi(\xi))$ expansion method [6,7], the Jacobi elliptic function expansion method [8], the generalized Riccati equation method $[9,10]$ the Sine-Cosine Method [11], the $F$-expansion method [12], and various other methods [13-16].

This paper presents an efficient generalized of $\cosh (\xi)$ expansion method for obtaining novel and more general exact traveling wave solutions for the derivative Schrödinger equation. The remaining of the paper is organized as follows. Section 2 explains the $\cosh (\xi)$ expansion method. Section 3 applies this method for solving derivative Schrödinger equation and presents some special solutions, which are shown graphically in Section 4. Section 5 concludes the paper.

\section{The Generalized of $\cosh (\xi)$ expansion method}

Suppose that we have a nonlinear PDE in the following form from the Introduction

$$
F\left(u, u_{t}, u_{x}, u_{t t}, u_{x t}, u_{x x}, u_{x x t}, \ldots\right)=0
$$

where, $u=u(x, t)$ is an unknown function, $\mathrm{F}$ is a polynomial in $\mathrm{u}=\mathrm{u}(\mathrm{x}, \mathrm{t})$ and its partial derivatives, in which the highest order derivatives and nonlinear terms are involved.The main steps of this method are as follows:

Step 1: Use the traveling wave transformation:

$$
u(x, t)=u(\xi), \quad \xi=k_{1} x+k_{2} t(2.2)
$$

where $k_{1}, k_{2}$ are a constants to be determined latter, permits us reducing (2.1) to an ODE for $u=u(\xi)$ in the form

$$
P\left(u, k_{1} u^{\prime}, k_{2} u^{\prime}, k_{1} k_{2} u^{\prime \prime}, \ldots\right)=0(2.3)
$$

where $P$ is a polynomial of $u=u(\xi)$ and its total derivatives.

Step 2:Balancing the highest derivative term with the nonlinear terms in (2.3), we find the value of the positive integer $(m)$.

Step 3:Suppose that the solution of (2.3) can be expressed as follows:

$$
u(\xi)=\alpha 0+\sum_{i=0}^{m}\left(\frac{A_{1} \operatorname{sech}(\xi)+A_{2} \cosh (\xi)+A_{3} \cosh ^{2}(\xi)+A_{4}}{B_{1} \operatorname{sech}(\xi)+B_{2} \cosh (\xi)+B_{3} \cosh ^{2}(\xi)+B_{4}}\right)^{i}
$$

where $\alpha_{0}, A_{i}, B_{i}(i=1,2,3,4)$ are constants to be determined later.

Step 4: Substituting (2.4) into Eq. (2.3) and then setting all the coefficients of $(\cosh (\xi))^{i}$ of the resulting systems to zero, yields a system of algebraic equations for $k_{1}, k_{2}, A_{i}, B_{i}(i=1,2,3,4)$ and $\alpha_{0}$. 
Step 5: Suppose that the value of the constants $k_{1}, k_{2}, A_{i}, B_{i}(i=1,2,3,4)$ and $\alpha_{0}$ can be found by solving the algebraic equations which are obtained in step 4. Since the general solutions of (2.3) have been well known for us, substituting $k_{1}, k_{2}, A_{i}, B_{i}(i=1,2,3,4)$ and $\alpha_{0}$ into (2.4), we have the exact solutions of the nonlinear PDEs (2.1).

\section{The Exact Solutions of Derivative Schrödinger Equation}

In this section, we will apply the the proposed method to find the exact solutions of the derivative Schrödinger equation. Let us consider the derivative Schrödinger equation:

$$
i W_{t}=\left(-\frac{1}{2}\right) W_{x x}+\left(i k|W|^{2} W\right)_{x} \quad ; \quad W=W(x, t)(3.1)
$$

we make the following transformation

$$
\begin{array}{lll}
W(x, t)=e^{(-i \delta t)} e^{i \psi(\xi)} h(\xi) ; & \xi=x-v t ; & \delta, v \in \Re \\
h, \psi: \Re^{2} \rightarrow \mathfrak{R} ; & h(\xi)>0
\end{array}
$$

Substituting (3.2) into Eq.(3.1) and making the real part and imaginary part equal to zero, we have

$$
\begin{gathered}
2 \delta h(\xi)+2 v h(\xi) \psi^{\prime}(\xi)+h^{\prime \prime}(\xi)-h(\xi) \psi^{\prime 2}(\xi)+2 k h^{3}(\xi) \psi^{\prime}(\xi)=0,(3.3) \\
h(\xi) \psi^{\prime \prime}(\xi)+2 h^{\prime}(\xi) \psi^{\prime}(\xi)-2 v h^{\prime}(\xi)-6 k h^{2}(\xi) h^{\prime}(\xi)=0
\end{gathered}
$$

Let

$$
\psi^{\prime}(\xi)=A+B h^{2}(\xi)(3.5)
$$

Substituting (3.5) into Eq. (3.4) and equating the coefficients of these terms $h^{\prime}(\xi) h^{2}(\xi), h^{\prime}(\xi)$ to zero, we get $A=v, B=\left(\frac{3 k}{2}\right)$. Therefore, when

$$
\psi^{\prime}(\xi)=v+\left(\frac{3 k}{2}\right) h^{2}(\xi)(3.6)
$$

Eq. (3.4) is identical to zero.

Substituting (3.6) into Eq. (3.3) we get the following equation:

$$
\left(2 \delta+v^{2}\right) h(\xi)+(2 v k) h^{3}(\xi)+\left(\frac{3 k^{2}}{4}\right) h^{5}(\xi)+h^{\prime \prime}(\xi)=0,(3.7)
$$

In order to solve Eq. (3.7), we make the following transformation

$$
h(\xi)=\sqrt{u(\xi)}(3.8)
$$

Then, $u(\xi)$ satisfies

$$
4\left(2 \delta+v^{2}\right) u^{2}+8 v k u^{3}+3 k^{2} u^{4}+2 u u^{\prime \prime}-u^{2}=0
$$

By balancing the term $\left(u^{2}\right)$ with the term $\left(u^{4}\right)$ in $(3.9)$, gives $(m=1)$. Therefore, the $\cosh (\xi)$ expansion method allows us to use the solution in the following form:

$$
u(\xi)=\alpha_{0}+\left(\frac{A_{1} \operatorname{sech}(\xi)+A_{2} \cosh (\xi)+A_{3} \cosh ^{2}(\xi)+A_{4}}{B_{1} \operatorname{sech}(\xi)+B_{2} \cosh (\xi)+B_{3} \cosh ^{2}(\xi)+B_{4}}\right)
$$

Substituting (3.10) into (3.9), the left-hand side is converted into polynomials in $(\cosh (\xi))^{j},(j=$ $0,1,2, \ldots .$. We collect each coefficient of these resulted polynomials to zero, yields a set of simultaneous algebraic equations (for simplicity, which are not presented) for $A_{1}, A_{2}, A_{3}, A_{4}, B_{1}, B_{2}, B_{3}, B_{4}, \delta, v$ and $\alpha_{0}$. Solving these algebraic equations with the help of algebraic software Maple, we obtain the following results: 
Case 1:

$$
\begin{aligned}
& \alpha_{0}=\alpha_{0}, v=-1, \delta=\frac{1}{8}, A_{1}=-\frac{1}{4} A_{2}, A_{2}=A_{2}, A_{3}=A_{2}\left(\alpha_{0} k-1\right) \\
& A_{4}=-\frac{1}{4} A_{3}, B_{1}=0, B_{2}=0, B_{3}=-k A_{2}, B_{4}=\frac{1}{4} k A_{2}
\end{aligned}
$$

Substituting (3.11) into (3.10), we have :

$$
u(\xi)=\frac{\cosh (\xi)-1}{k \cosh (\xi)}(3.12)
$$

where $\quad \xi=x+t$

Substituting (3.12) into (3.8) and (3.6) yields

$$
\begin{aligned}
& h(\xi)=\sqrt{\frac{\cosh (\xi)-1}{k \cosh (\xi)}} \\
& \psi(\xi)=\left(-1+\left(\frac{3 k}{2}\right)\left(\frac{\cosh (\xi)-1}{k \cosh (\xi)}\right)\right) d \xi \\
& =\left(\frac{3}{2} k \alpha_{0}-1\right) \xi+\frac{3}{2}\left(k \alpha_{0}+1\right) \ln \left(\frac{\tanh \left(\frac{\xi}{2}\right)+1}{\tanh \left(\frac{\xi}{2}\right)-1}\right)-3 \arctan \left(\tanh \left(\frac{\xi}{2}\right)\right)
\end{aligned}
$$

Consequently, the exact solution of the derivative Schrödinger equation (3.1) with the help of Eq. (3.13) and Eq. (3.2) are obtained in the following form:

$$
W(x, t)=\left(\sqrt{\frac{\cosh (\xi)-1}{k \cosh (\xi)}}\right) \exp \left(i\left(\begin{array}{l}
\left(\frac{3}{2} k \alpha_{0}-1\right) \xi \\
+\frac{3}{2}\left(k \alpha_{0}+1\right) \ln \left(\frac{\tanh \left(\frac{\xi}{2}\right)+1}{\tanh \left(\frac{\xi}{2}\right)-1}\right) \\
\xi=x+t
\end{array}\right)\right)
$$

In particular setting $\quad \alpha_{0}=0, k=6$ we find :

$$
\begin{aligned}
& W_{1}(x, t)=\left(\sqrt{\frac{\cosh (\xi)-1}{6 \cosh (\xi)}}\right) \exp \left(i\left(\begin{array}{l}
-\xi+\frac{3}{2} \ln \left(\frac{\tanh \left(\frac{\xi}{2}\right)+1}{\tanh \left(\frac{\xi}{2}\right)-1}\right) \\
-3 \arctan \left(\tanh \left(\frac{\xi}{2}\right)\right)-\frac{1}{8} t
\end{array}\right)\right) \\
& \xi=x+t
\end{aligned}
$$

See Figure (3.1)

\section{Case 2:}

$$
\begin{aligned}
& \alpha_{0}=\alpha_{0}, v=-2, \delta=\frac{1}{2}, A_{1}=\frac{B_{1}\left(4-\alpha_{0} k\right)}{k}, A_{2}=\frac{2 B_{1}\left(\alpha_{0} k-2\right)}{k} \\
& A_{3}=0, A_{4}=0, B_{1}=B_{1}, B_{2}=-2 B_{1}, B_{3}=0, B_{4}=0
\end{aligned}
$$

Substituting (3.16) into(3.10), we have : 
where $\quad \xi=x+2 t$

$$
u(\xi)=\frac{4(\operatorname{sech}(\xi)-\cosh (\xi))}{k(\operatorname{sech}(\xi)-2 \cosh (\xi))}(3.17)
$$

Substituting (3.17) into (3.8) and (3.6) yields

$$
\begin{aligned}
h(\xi) & =\sqrt{\left(\frac{4}{k}\right)\left(\frac{\operatorname{sech}(\xi)-\cosh (\xi)}{\operatorname{sech}(\xi)-2 \cosh (\xi)}\right)} \\
\psi(\xi) & =\left(-2+6\left(\frac{\operatorname{sech}(\xi)-\cosh (\xi)}{\operatorname{sech}(\xi)-2 \cosh (\xi)}\right)\right) d \xi \\
= & \left(\begin{array}{l}
\left(\frac{3}{2} \alpha_{0} k-2\right) \xi+\left(\frac{3}{2} \alpha_{0} k-3\right) \ln \left(\frac{\tanh \left(\frac{\xi}{2}\right)-1}{\tanh \left(\frac{\xi}{2}\right)+1}\right) \\
+3 \arctan \left(\frac{\tanh \left(\frac{\xi}{2}\right)}{\sqrt{2}+1}\right)+3 \arctan \left(\frac{\tanh \left(\frac{\xi}{2}\right)}{\sqrt{2}-1}\right)
\end{array}\right)
\end{aligned}
$$

Consequently,the exact solution of the derivative Schrödinger equation (3.1) with the help of Eq. (3.18) and Eq. (3.2) are obtained in the following form:

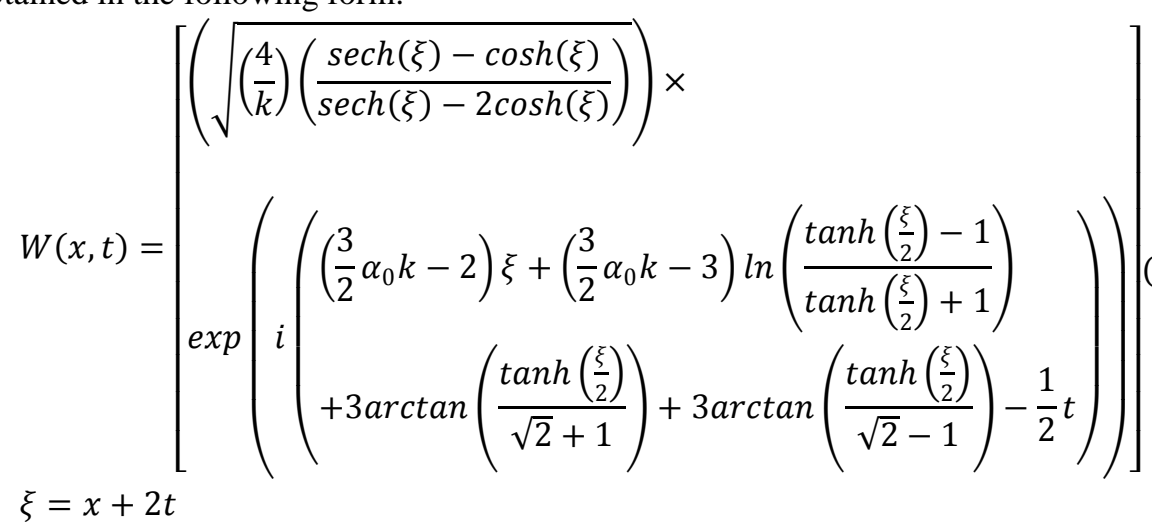

In particular setting $\quad \alpha_{0}=0, k=4$ we find :

$$
\begin{aligned}
& \left.W_{2}(x, t)=\left[\begin{array}{l}
\left(\sqrt{\left(\frac{\operatorname{sech}(\xi)-\cosh (\xi)}{\operatorname{sech}(\xi)-2 \cosh (\xi)}\right)}\right) \times \\
\exp \left(\begin{array}{l}
\left.-3 \ln \left(\frac{\tanh \left(\frac{\xi}{2}\right)-1}{\tanh \left(\frac{\xi}{2}\right)+1}\right)+3 \arctan \left(\frac{\tanh \left(\frac{\xi}{2}\right)}{\sqrt{2}+1}\right)\right) \\
+3 \arctan \left(\frac{\tanh \left(\frac{\xi}{2}\right)}{\sqrt{2}-1}\right)-2 \xi-\frac{1}{2} t
\end{array}\right)
\end{array}\right)\right] \\
& \xi=x+2 t
\end{aligned}
$$

See Figure (3 .2)

Case 3:

$$
\begin{aligned}
& \alpha_{0}=\alpha_{0}, v=\frac{1+3 \alpha_{0}^{2} k^{2}}{-4 \alpha_{0} k}, \delta=\frac{\left(\alpha_{0}^{2} k^{2}+1\right)\left(3 \alpha_{0}^{2} k^{2}-1\right)}{32 \alpha_{0}^{2} k^{2}}, A_{1}=A_{1}, A_{2}=A_{2}, A_{3}=0 \\
& A_{4}=A_{4}, B_{1}=\frac{\left(\alpha_{0}^{2} k^{2}-1\right) A_{2}}{2 \alpha_{0}}, B_{2}=\frac{A_{4}\left(\alpha_{0}^{2} k^{2}+1\right)+A_{2}\left(\alpha_{0}^{2} k^{2}-1\right)}{2 \alpha_{0}}, B_{3}=\frac{\left(\alpha_{0}^{2} k^{2}+1\right) A_{2}}{2 \alpha_{0}},(3.21) \\
& B_{4}=\frac{A_{4}\left(\alpha_{0}^{2} k^{2}-1\right)+A_{1}\left(\alpha_{0}^{2} k^{2}+1\right)}{2 \alpha_{0}}
\end{aligned}
$$


Substituting (3.21) into(3.10), we have :

$$
u(\xi)=\alpha_{0}\left(\frac{\left(\alpha_{0}^{2} k^{2}+1\right) \cosh (\xi)+\left(\alpha_{0}^{2} k^{2}+1\right)}{\left(\alpha_{0}^{2} k^{2}+1\right) \cosh (\xi)+\left(\alpha_{0}^{2} k^{2}-1\right)}\right)
$$

where $\quad \xi=x+\left(\frac{1+3 \alpha_{0}^{2} k^{2}}{4 \alpha_{0} k}\right) t$

Substituting (3.22) into (3.8) and (3.6) yields

$$
\begin{aligned}
& h(\xi)=\sqrt{\alpha_{0}\left(\frac{\left(\alpha_{0}^{2} k^{2}+1\right) \cosh (\xi)+\left(\alpha_{0}^{2} k^{2}+1\right)}{\left(\alpha_{0}^{2} k^{2}+1\right) \cosh (\xi)+\left(\alpha_{0}^{2} k^{2}-1\right)}\right)} \\
& \psi(\xi)=\left(v+\left(\frac{3 k \alpha_{0}}{2}\right)\left(\frac{\left(\alpha_{0}^{2} k^{2}+1\right) \cosh (\xi)+\left(\alpha_{0}^{2} k^{2}+1\right)}{\left(\alpha_{0}^{2} k^{2}+1\right) \cosh (\xi)+\left(\alpha_{0}^{2} k^{2}-1\right)}\right)\right) d \xi(3.23) \\
& =\left(\frac{3}{4} \alpha_{0} k-\frac{1}{4 \alpha_{0} k}\right) \xi+3 \arctan \left(\frac{\tan \left(\frac{\xi}{2}\right)}{\alpha_{0} k}\right)
\end{aligned}
$$

Consequently,the exact solution of the derivative Schrödinger equation (3.1) with the help of Eq. (3.23) and Eq (3.2) are obtained in the following form:

$$
\left.W(x, t)=\left[\begin{array}{l}
\left(\sqrt{\alpha_{0}\left(\frac{\left(\alpha_{0}^{2} k^{2}+1\right) \cosh (\xi)+\left(\alpha_{0}^{2} k^{2}+1\right)}{\left(\alpha_{0}^{2} k^{2}+1\right) \cosh (\xi)+\left(\alpha_{0}^{2} k^{2}-1\right)}\right)}\right) \times \\
\left.\exp \left(\begin{array}{l}
\left.\left(\frac{3}{4} \alpha_{0} k-\frac{1}{4 \alpha_{0} k}\right) \xi+3 \arctan \left(\frac{\tan \left(\frac{\xi}{2}\right)}{\alpha_{0} k}\right)\right) \\
-\left(\frac{\left(\alpha_{0}^{2} k^{2}+1\right)\left(3 \alpha_{0}^{2} k^{2}-1\right)}{32 \alpha_{0}^{2} k^{2}}\right) t
\end{array}\right)\right)
\end{array}\right)\right]
$$

In particular setting $\quad \alpha_{0}=1, k=1$ we find :

$$
\begin{aligned}
& W_{3}(x, t)=\left(\sqrt{\frac{\cosh (\xi)+1}{\cosh (\xi)}}\right) \exp \left(i\left(\frac{1}{2} \xi+3 \arctan \left(\tan \left(\frac{\xi}{2}\right)\right)-\frac{1}{8} t\right)\right) \\
& \xi=x+t
\end{aligned}
$$

See Figure (3.3)

\section{Case 4:}

$$
\begin{aligned}
& \alpha_{0}=\alpha_{0}, v=v, \delta=-\left(\frac{9}{8}+\frac{v^{2}}{2}\right), A_{1}=-\frac{B_{4}\left(2 \alpha_{0} v k-9\right)}{3 k \sqrt{4 v^{2}+9}}, A_{2}=0, A_{3}=\frac{4}{3} \alpha_{0} B_{4} \\
& A_{4}=-\alpha_{0} B_{4}, B_{1}=\frac{2 v B_{4}}{3 \sqrt{4 v^{2}+9}}, B_{2}=0, B_{3}=-\frac{4}{3} B_{4}, B_{4}=B_{4}
\end{aligned}
$$

Substituting (3.26) into(3.10), we have :

$$
u(\xi)=\frac{9 \operatorname{sech}(\xi)}{(2 v k) \operatorname{sech}(\xi)-\left(4 k \sqrt{4 v^{2}+9}\right) \cosh ^{2}(\xi)+\left(3 k \sqrt{4 v^{2}+9}\right)}
$$

where $\quad \xi=x-v t$

Substituting (3.27) into (3.8) and (3.6) yields 


$$
\begin{aligned}
& h(\xi)=\sqrt{\frac{9 \operatorname{sech}(\xi)}{(2 v k) \operatorname{sech}(\xi)-\left(4 k \sqrt{4 v^{2}+9}\right) \cosh ^{2}(\xi)+\left(3 k \sqrt{4 v^{2}+9}\right)}} \\
& \psi(\xi)=\left(v+\left(\frac{3 k}{2}\right)\left(\frac{9 \operatorname{sech}(\xi)}{(2 v k) \operatorname{sech}(\xi)-\left(4 k \sqrt{4 v^{2}+9}\right) \cosh ^{2}(\xi)+\left(3 k \sqrt{4 v^{2}+9}\right)}\right)\right) d \xi \\
& =\left[v \xi+(27 k) \sum_{\varepsilon}\left(\frac{\left(\varepsilon^{4}-2 \varepsilon^{2}+1\right) \ln \left(\tanh \left(\frac{\xi}{2}\right)-\varepsilon\right)}{6\left(S_{3}+S_{2}-S_{1}\right) \varepsilon^{5}+4\left(3 S_{1}+3 S_{2}-S_{3}\right) \varepsilon^{3}+2\left(3 S_{2}-3 S_{1}-S_{3}\right) \varepsilon}\right)\right] \\
& S_{1}=(2 v k), S_{2}=-\left(4 k \sqrt{4 v^{2}+9}\right), S_{3}=\left(3 k \sqrt{4 v^{2}+9}\right) \\
& 0=\left(S_{2}+S_{3}-S_{1}\right) \varepsilon^{6}+\left(3 S_{1}+3 S_{2}-S_{3}\right) \varepsilon^{4}+\left(3 S_{2}-3 S_{1}-S_{3}\right) \varepsilon^{2}+\left(S_{1}+S_{2}+S_{3}\right)
\end{aligned}
$$

Consequently,the exact solution of the derivative Schrödinger equation (3.1) with the help of Eq. (3.28) and Eq. (3.2) are obtained in the following form:

$$
\begin{aligned}
& W(x, t)=e^{\left(\frac{9}{8}+\frac{v^{2}}{2}\right) i t} e^{i \psi(\xi)} \sqrt{\frac{9 \operatorname{sech}(\xi)}{(2 v k) \operatorname{sech}(\xi)-\left(4 k \sqrt{4 v^{2}+9}\right) \cosh ^{2}(\xi)+\left(3 k \sqrt{4 v^{2}+9}\right)}(3.29)} \\
& \xi=x-v t
\end{aligned}
$$

In particular setting $\quad \alpha_{0}=0, v=0, k=-1$ we find :

$$
W_{4}(x, t)=\left[\begin{array}{l}
\left(\sqrt{\frac{3 \operatorname{sech}(\xi)}{4 \cosh ^{2}(\xi)-3}}\right) \times \\
\xi=x
\end{array}\right.
$$

See Figure (3 .4)

\section{Case 5:}

$$
\begin{aligned}
& \alpha_{0}=\alpha_{0}, v=-\frac{3 k^{2}\left(\alpha_{0} B_{2}+A_{2}\right)^{2}+4 B_{2}^{2}}{4 k B_{2}\left(\alpha_{0} B_{2}+A_{2}\right)}, A_{2}=A_{2}, A_{3}=0, A_{4}=0 \\
& A_{1}=\frac{4 \alpha_{0} B_{2}^{3}}{k^{2}\left(\alpha_{0} B_{2}+A_{2}\right)^{2}+4 B_{2}^{2}}, \delta=\frac{\left(3 k^{2}\left(\alpha_{0} B_{2}+A_{2}\right)^{2}-4 B_{2}^{2}\right)\left(k^{2}\left(\alpha_{0} B_{2}+A_{2}\right)^{2}+4 B_{2}^{2}\right)}{32 k^{2} B_{2}^{2}\left(\alpha_{0} B_{2}+A_{2}\right)^{2}},(3 \\
& B_{1}=-\frac{4 B_{2}^{3}}{k^{2}\left(\alpha_{0} B_{2}+A_{2}\right)^{2}+4 B_{2}^{2}}, B_{2}=B_{2}, B_{3}=0, B_{4}=0
\end{aligned}
$$

Substituting (3.31) into(3.10), we have :

$$
u(\xi)=\frac{\left(\alpha_{0} B_{2}+A_{2}\right)\left(k^{2}\left(\alpha_{0} B_{2}+A_{2}\right)^{2}+4 B_{2}^{2}\right) \cosh (\xi)}{\left(-4 B_{2}^{3}\right) \operatorname{sech}(\xi)+\left(k^{2} B_{2}\left(\alpha_{0} B_{2}+A_{2}\right)^{2}+4 B_{2}^{3}\right) \cosh (\xi)}
$$

where $\quad \xi=x+\left(\frac{3 k^{2}\left(\alpha_{0} B_{2}+A_{2}\right)^{2}+4 B_{2}^{2}}{4 k B_{2}\left(\alpha_{0} B_{2}+A_{2}\right)}\right) t$

Substituting (3.32) into (3.8) and (3.6) yields 


$$
\begin{aligned}
& h(\xi)=\sqrt{\frac{\left(\alpha_{0} B_{2}+A_{2}\right)\left(k^{2}\left(\alpha_{0} B_{2}+A_{2}\right)^{2}+4 B_{2}^{2}\right) \cosh (\xi)}{\left(-4 B_{2}^{3}\right) \operatorname{sech}(\xi)+\left(k^{2} B_{2}\left(\alpha_{0} B_{2}+A_{2}\right)^{2}+4 B_{2}^{3}\right) \cosh (\xi)}} \\
& \psi(\xi)=\left(v+\left(\frac{3 k}{2}\right)\left(\frac{\left(\alpha_{0} B_{2}+A_{2}\right)\left(k^{2}\left(\alpha_{0} B_{2}+A_{2}\right)^{2}+4 B_{2}^{2}\right) \cosh (\xi)}{\left(-4 B_{2}^{3}\right) \operatorname{sech}(\xi)+\left(k^{2} B_{2}\left(\alpha_{0} B_{2}+A_{2}\right)^{2}+4 B_{2}^{3}\right) \cosh (\xi)}\right)\right) d \xi \\
& =\left(\begin{array}{l}
\left(\frac{3 k L_{1}}{4 L_{3}}\right) \sqrt{\frac{L_{2}}{L_{3}+L_{2}}} \ln \left(\frac{\sqrt{L_{3}+L_{2}} \tanh ^{2}\left(\frac{\xi}{2}\right)-2 \sqrt{L_{2}} \tanh \left(\frac{\xi}{2}\right)+\sqrt{L_{3}+L_{2}}}{\sqrt{L_{3}+L_{2}} \tanh ^{2}\left(\frac{\xi}{2}\right)+2 \sqrt{L_{2}} \tanh \left(\frac{\xi}{2}\right)+\sqrt{L_{3}+L_{2}}}\right) \\
+v \xi+\left(\frac{3 k L_{1}}{2 L_{3}}\right) \ln \left(\frac{\tanh \left(\frac{\xi}{2}\right)+1}{\tanh \left(\frac{\xi}{2}\right)-1}\right)
\end{array}\right) \\
& L_{1}=\left(\alpha_{0} B_{2}+A_{2}\right)\left(k^{2}\left(\alpha_{0} B_{2}+A_{2}\right)^{2}+4 B_{2}^{2}\right) ; L_{2}=\left(-4 B_{2}^{3}\right) ; L_{3}=\left(k^{2} B_{2}\left(\alpha_{0} B_{2}+A_{2}\right)^{2}+4 B_{2}^{3}\right)
\end{aligned}
$$

Consequently,the exact solution of the derivative Schrödinger equation (3.1) with the help of Eq. (3.33) and Eq. (3.2) are obtained in the following form:

$$
\begin{aligned}
& \left.W(x, t)=\left[\begin{array}{l}
\left(\frac{\left(\alpha_{0} B_{2}+A_{2}\right)\left(k^{2}\left(\alpha_{0} B_{2}+A_{2}\right)^{2}+4 B_{2}^{2}\right) \cosh (\xi)}{\left(-4 B_{2}^{3}\right) \operatorname{sech}(\xi)+\left(k^{2} B_{2}\left(\alpha_{0} B_{2}+A_{2}\right)^{2}+4 B_{2}^{3}\right) \cosh (\xi)}\right) \times \\
\left.\exp \left(\begin{array}{l}
\left(\frac{3 k L_{1}}{2 L_{3}}\right) \ln \left(\frac{\tanh \left(\frac{\xi}{2}\right)+1}{\tanh \left(\frac{\xi}{2}\right)-1}\right)-\left(\frac{\left(3 k^{2}\left(\alpha_{0} B_{2}+A_{2}\right)^{2}-4 B_{2}^{2}\right)\left(k^{2}\left(\alpha_{0} B_{2}+A_{2}\right)^{2}+4 B_{2}^{2}\right)}{32 k^{2} B_{2}^{2}\left(\alpha_{0} B_{2}+A_{2}\right)^{2}}\right) t \\
+\left(\frac{3 k L_{1}}{4 L_{3}}\right) \sqrt{\frac{L_{2}}{L_{3}+L_{2}}} \ln \left(\frac{\sqrt{L_{3}+L_{2}} \tanh ^{2}\left(\frac{\xi}{2}\right)-2 \sqrt{L_{2}} \tanh \left(\frac{\xi}{2}\right)+\sqrt{L_{3}+L_{2}}}{\sqrt{L_{3}+L_{2}} \tanh ^{2}\left(\frac{\xi}{2}\right)+2 \sqrt{L_{2}} \tanh \left(\frac{\xi}{2}\right)+\sqrt{L_{3}+L_{2}}}\right)+v \xi
\end{array}\right)\right)
\end{array}\right)\right] \\
& \xi=x+\left(\frac{3 k^{2}\left(\alpha_{0} B_{2}+A_{2}\right)^{2}+4 B_{2}^{2}}{4 k B_{2}\left(\alpha_{0} B_{2}+A_{2}\right)}\right) t
\end{aligned}
$$

In particular setting $\alpha_{0}=1, k=2, A_{2}=2, B_{2}=1$ we find :

$$
\begin{aligned}
& W_{5}(x, t)=\left(\sqrt{\frac{30 \cosh (\xi)}{10 \cosh (\xi)-\operatorname{sech}(\xi)}}\right) \times \exp \left(i\left(\begin{array}{c}
6 \ln \left(\frac{\tanh \left(\frac{\xi}{2}\right)+1}{\tanh \left(\frac{\xi}{2}\right)-1}\right)+3 \arctan \left(\frac{3 \tanh \left(\frac{\xi}{2}\right)}{\sqrt{10}-1}\right) \\
-3 \arctan \left(\frac{3 \tanh \left(\frac{\xi}{2}\right)}{\sqrt{10}+1}\right)-\frac{5}{3} \xi-\left(\frac{65}{18}\right) t
\end{array}\right)\right) \\
& \xi=x+\left(\frac{14}{3}\right) t
\end{aligned}
$$

See Figure (3 .5)

Case 6

$$
\begin{aligned}
& v=-\frac{3 k^{2}\left(\alpha_{0} B_{3}+A_{3}\right)^{2}+4 B_{3}^{2}}{4 k B_{3}\left(\alpha_{0} B_{3}+A_{3}\right)}, \delta=\frac{\left(k^{2}\left(\alpha_{0} B_{3}+A_{3}\right)^{2}+4 B_{3}^{2}\right)\left(3 k^{2}\left(\alpha_{0} B_{3}+A_{3}\right)^{2}-4 B_{3}^{2}\right)}{32 k^{2}\left(\alpha_{0} B_{3}+A_{3}\right)^{2} B_{3}^{2}}, \\
& A_{1}=\frac{4 \alpha_{0} B_{2} B_{3}^{2}}{k^{2}\left(\alpha_{0} B_{3}+A_{3}\right)^{2}+4 B_{3}^{2}}, A_{2}=\frac{A_{3} B_{2}}{B_{3}}, A_{3}=A_{3}, A_{4}=\frac{4 \alpha_{0} B_{3}^{3}}{k^{2}\left(\alpha_{0} B_{3}+A_{3}\right)^{2}+4 B_{3}^{2}}, \alpha_{0}=\alpha_{0} \\
& B_{1}=\frac{-4 B_{2} B_{3}^{2}}{k^{2}\left(\alpha_{0} B_{3}+A_{3}\right)^{2}+4 B_{3}^{2}}, B_{2}=B_{2}, B_{3}=B_{3}, B_{4}=\frac{-4 B_{3}^{3}}{k^{2}\left(\alpha_{0} B_{3}+A_{3}\right)^{2}+4 B_{3}^{2}}
\end{aligned}
$$

Substituting (3.36) into(3.10), we have :

$$
u(\xi)=\frac{\left(\alpha_{0} B_{3}+A_{3}\right)\left(k^{2}\left(\alpha_{0} B_{3}+A_{3}\right)^{2}+4 B_{3}^{2}\right) \cosh ^{2}(\xi)}{\left(-4 B_{2}^{3}\right)+\left(k^{2} B_{3}\left(\alpha_{0} B_{3}+A_{3}\right)^{2}+4 B_{3}^{3}\right) \cosh ^{2}(\xi)}
$$

where $\quad \xi=x+\left(\frac{3 k^{2}\left(\alpha_{0} B_{3}+A_{3}\right)^{2}+4 B_{3}^{2}}{4 k B_{3}\left(\alpha_{0} B_{3}+A_{3}\right)}\right) t$ 
Substituting (3.37) into (3.8) and (3.6) yields

$$
\begin{aligned}
& h(\xi)=\sqrt{\frac{\left(\alpha_{0} B_{3}+A_{3}\right)\left(k^{2}\left(\alpha_{0} B_{3}+A_{3}\right)^{2}+4 B_{3}^{2}\right) \cosh ^{2}(\xi)}{\left(-4 B_{2}^{3}\right)+\left(k^{2} B_{3}\left(\alpha_{0} B_{3}+A_{3}\right)^{2}+4 B_{3}^{3}\right) \cosh ^{2}(\xi)}} \\
& \psi(\xi)=\left(v+\left(\frac{3 k}{2}\right)\left(\frac{\left(\alpha_{0} B_{3}+A_{3}\right)\left(k^{2}\left(\alpha_{0} B_{3}+A_{3}\right)^{2}+4 B_{3}^{2}\right) \cosh ^{2}(\xi)}{\left(-4 B_{2}^{3}\right)+\left(k^{2} B_{3}\left(\alpha_{0} B_{3}+A_{3}\right)^{2}+4 B_{3}^{3}\right) \cosh ^{2}(\xi)}\right)\right) d \xi \\
&=\left(\begin{array}{l}
\left(\frac{3 k T_{1} \sqrt{T_{2}}}{4 T_{3} \sqrt{T_{2}+T_{3}}}\right) \ln \left(\frac{\sqrt{T_{2}+T_{3}} \tanh ^{2}\left(\frac{\xi}{2}\right)-2 \sqrt{T_{2}} \tanh \left(\frac{\xi}{2}\right)+\sqrt{T_{2}+T_{3}}}{\sqrt{T_{2}+T_{3}} \tanh ^{2}\left(\frac{\xi}{2}\right)+2 \sqrt{T_{2}} \tanh \left(\frac{\xi}{2}\right)+\sqrt{T_{2}+T_{3}}}\right)+ \\
+\left(\frac{3 k T_{1}}{4 T_{3}}\right) \ln \left(\frac{\tanh \left(\frac{\xi}{2}\right)+1}{\tanh \left(\frac{\xi}{2}\right)-1}\right)+v \xi
\end{array}\right) \\
& T_{1}=\left(\alpha_{0} B_{3}+A_{3}\right)\left(k^{2}\left(\alpha_{0} B_{3}+A_{3}\right)^{2}+4 B_{3}^{2}\right), T_{2}=\left(-4 B_{2}^{3}\right) \\
& T_{3}=\left(k^{2} B_{3}\left(\alpha_{0} B_{3}+A_{3}\right)^{2}+4 B_{3}^{3}\right)
\end{aligned}
$$

Consequently,the exact solution of the derivative Schrödinger equation (3.1) with the help of Eq. (3.38) and Eq. (3.2) are obtained in the following form:

$$
\left.W(x, t)=\left[\begin{array}{l}
\left(\begin{array}{l}
\left.\frac{\left(\alpha_{0} B_{3}+A_{3}\right)\left(k^{2}\left(\alpha_{0} B_{3}+A_{3}\right)^{2}+4 B_{3}^{2}\right) \cosh ^{2}(\xi)}{\left(-4 B_{2}^{3}\right)+\left(k^{2} B_{3}\left(\alpha_{0} B_{3}+A_{3}\right)^{2}+4 B_{3}^{3}\right) \cosh ^{2}(\xi)}\right) \times \\
\left.\exp \left(\begin{array}{l}
\left(\frac{3 k T_{1} \sqrt{T_{2}}}{4 T_{3} \sqrt{T_{2}+T_{3}}}\right) \ln \left(\frac{\sqrt{T_{2}+T_{3}} \tanh ^{2}\left(\frac{\xi}{2}\right)-2 \sqrt{T_{2}} \tanh \left(\frac{\xi}{2}\right)+\sqrt{T_{2}+T_{3}}}{\sqrt{T_{2}+T_{3}} \tanh ^{2}\left(\frac{\xi}{2}\right)+2 \sqrt{T_{2}} \tanh \left(\frac{\xi}{2}\right)+\sqrt{T_{2}+T_{3}}}\right)+v \xi \\
+\left(\frac{3 k T_{1}}{4 T_{3}}\right) \ln \left(\frac{\tanh \left(\frac{\xi}{2}\right)+1}{\tanh \left(\frac{\xi}{2}\right)-1}\right)-\left(\frac{\left(k^{2}\left(\alpha_{0} B_{3}+A_{3}\right)^{2}+4 B_{3}^{2}\right)\left(3 k^{2}\left(\alpha_{0} B_{3}+A_{3}\right)^{2}-4 B_{3}^{2}\right)}{32 k^{2}\left(\alpha_{0} B_{3}+A_{3}\right)^{2} B_{3}^{2}}\right) t
\end{array}\right)\right)
\end{array}\right) \\
\xi=x+\left(\frac{3 k^{2}\left(\alpha_{0} B_{3}+A_{3}\right)^{2}+4 B_{3}^{2}}{4 k B_{3}\left(\alpha_{0} B_{3}+A_{3}\right)}\right) t
\end{array}\right)\right]
$$

In particular setting $\quad \alpha_{0}=0, k=1, A_{3}=3, B_{3}=3$ we find :

$$
\begin{aligned}
& W_{6}(x, t)=\left(\sqrt{\frac{5 \cosh ^{2}(\xi)}{5 \cosh ^{2}(\xi)-4}}\right) \times \exp \left(i\left(\begin{array}{l}
3 \arctan \left(\frac{\tanh \left(\frac{\xi}{2}\right)}{\sqrt{5}-2}\right)-3 \arctan \left(\frac{\tanh \left(\frac{\xi}{2}\right)}{\sqrt{5}+2}\right) \\
+\left(\frac{3}{2}\right) \ln \left(\frac{\tanh \left(\frac{\xi}{2}\right)+1}{\tanh \left(\frac{\xi}{2}\right)-1}\right)-\frac{7}{4} \xi+\left(\frac{5}{32}\right) t
\end{array}\right)\right) \\
& \xi=x+\frac{7}{4} t
\end{aligned}
$$

See Figure (3.6)

Case 7:

$$
\begin{aligned}
& \alpha_{0}=\alpha_{0}, v=-\frac{k^{2}\left(A_{1}+\alpha_{0} B_{1}\right)^{2}-4 B_{1}^{2}}{4 k B_{1}\left(A_{1}+\alpha_{0} B_{1}\right)}, \delta=-\frac{\left(k^{2}\left(A_{1}+\alpha_{0} B_{1}\right)^{2}+4 B_{1}^{2}\right)^{2}}{32 k^{2} B_{1}^{2}\left(A_{1}+\alpha_{0} B_{1}\right)^{2}}, \\
& A_{1}=A_{1}, A_{2}=\frac{\alpha_{0}\left(k^{2}\left(A_{1}+\alpha_{0} B_{1}\right)^{2}+4 B_{1}^{2}\right)}{4 B_{1}}, A_{3}=0, A_{4}=0 \\
& B_{1}=B_{1}, B_{2}=-\frac{\alpha_{0}\left(k^{2}\left(A_{1}+\alpha_{0} B_{1}\right)^{2}+4 B_{1}^{2}\right)}{4 B_{1}}, B_{3}=0, B_{4}=0
\end{aligned}
$$


Substituting (3.41) into(3.10), we have :

$$
u(\xi)=\frac{4 B_{1}\left(\alpha_{0} B_{1}+A_{1}\right) \operatorname{sech}(\xi)}{\left(4 B_{1}^{2}\right) \operatorname{sech}(\xi)-\left(k^{2}\left(\alpha_{0} B_{1}+A_{1}\right)^{2}+4 B_{1}^{2}\right) \cosh (\xi)}
$$

where $\quad \xi=x+\left(\frac{k^{2}\left(A_{1}+\alpha_{0} B_{1}\right)^{2}-4 B_{1}^{2}}{4 k B_{1}\left(A_{1}+\alpha_{0} B_{1}\right)}\right) t$

Substituting (3.42) into (3.8) and (3.6) yields

$$
\begin{aligned}
& h(\xi)=\sqrt{\frac{4 B_{1}\left(\alpha_{0} B_{1}+A_{1}\right) \operatorname{sech}(\xi)}{\left(4 B_{1}^{2}\right) \operatorname{sech}(\xi)-\left(k^{2}\left(\alpha_{0} B_{1}+A_{1}\right)^{2}+4 B_{1}^{2}\right) \cosh (\xi)}} \\
& \begin{array}{l}
\psi(\xi)=\left(v+\left(\frac{3 k}{2}\right)\left(\frac{4 B_{1}\left(\alpha_{0} B_{1}+A_{1}\right) \operatorname{sech}(\xi)}{\left(4 B_{1}^{2}\right) \operatorname{sech}(\xi)-\left(k^{2}\left(\alpha_{0} B_{1}+A_{1}\right)^{2}+4 B_{1}^{2}\right) \cosh (\xi)}\right)\right) d \xi \\
=v \xi+\left(\frac{3 k \beta_{1}}{4 \sqrt{\beta_{2}} \sqrt{\beta_{2}+\beta_{3}}}\right) \ln \left(\frac{\sqrt{\beta_{2}+\beta_{3}} \tanh ^{2}\left(\frac{\xi}{2}\right)+2 \sqrt{\beta_{2}} \tanh \left(\frac{\xi}{2}\right)+\sqrt{\beta_{2}+\beta_{3}}}{\sqrt{\beta_{2}+\beta_{3}} \tanh ^{2}\left(\frac{\xi}{2}\right)-2 \sqrt{\beta_{2}} \tanh \left(\frac{\xi}{2}\right)+\sqrt{\beta_{2}+\beta_{3}}}\right) \\
\beta_{1}=4 B_{1}\left(\alpha_{0} B_{1}+A_{1}\right), \beta_{2}=\left(4 B_{1}^{2}\right), \beta_{3}=\left(k^{2}\left(\alpha_{0} B_{1}+A_{1}\right)^{2}+4 B_{1}^{2}\right)
\end{array}
\end{aligned}
$$

Consequently,the exact solution of the derivative Schrödinger equation (3.1) with the help of Eq. (3.43) and Eq. (3.2) are obtained in the following form:

$$
\left.W(x, t)=\left[\begin{array}{l}
\left(\sqrt{\frac{4 B_{1}\left(\alpha_{0} B_{1}+A_{1}\right) \operatorname{sech}(\xi)}{\left(4 B_{1}^{2}\right) \operatorname{sech}(\xi)-\left(k^{2}\left(\alpha_{0} B_{1}+A_{1}\right)^{2}+4 B_{1}^{2}\right) \cosh (\xi)}}\right) \times \\
\left.\exp \left(\begin{array}{l}
\left.\left(\frac{3 k \beta_{1}}{4 \sqrt{\beta_{2}} \sqrt{\beta_{2}+\beta_{3}}}\right) \ln \left(\frac{\sqrt{\beta_{2}+\beta_{3}} \tanh ^{2}\left(\frac{\xi}{2}\right)+2 \sqrt{\beta_{2}} \tanh \left(\frac{\xi}{2}\right)+\sqrt{\beta_{2}+\beta_{3}}}{\sqrt{\beta_{2}+\beta_{3}} \tanh ^{2}\left(\frac{\xi}{2}\right)-2 \sqrt{\beta_{2}} \tanh \left(\frac{\xi}{2}\right)+\sqrt{\beta_{2}+\beta_{3}}}\right)\right) \\
+v \xi+\left(\frac{\left(k^{2}\left(A_{1}+\alpha_{0} B_{1}\right)^{2}+4 B_{1}^{2}\right)^{2}}{32 k^{2} B_{1}^{2}\left(A_{1}+\alpha_{0} B_{1}\right)^{2}}\right) t
\end{array}\right)\right)
\end{array}\right)\right]
$$

In particular setting $\alpha_{0}=1, k=6, A_{1}=1, B_{1}=1$ we find :

$$
\begin{aligned}
& W_{7}(x, t)=\left(\sqrt{\frac{8 \operatorname{sech}(\xi)}{4 \operatorname{sech}(\xi)-148 \cosh (\xi)}}\right) \exp \left(i\left(\begin{array}{l}
\left(-\frac{35}{12}\right) \xi+3 \arctan \left(\frac{6 \tanh \left(\frac{\xi}{2}\right)}{\sqrt{37}+1}\right) \\
-3 \arctan \left(\frac{6 \tanh \left(\frac{\xi}{2}\right)}{\sqrt{37}-1}\right)+\left(\frac{1369}{288}\right) t
\end{array}\right)\right)(3.4 \\
& \xi=x+\left(\frac{35}{12}\right) t
\end{aligned}
$$

See Figure (3.7)

Case 8:

$$
\begin{aligned}
& \alpha_{0}=\alpha_{0}, v=-\frac{3 k^{2}\left(A_{4}+\alpha_{0} B_{4}\right)^{2}+B_{4}^{2}}{4 k B_{4}\left(A_{4}+\alpha_{0} B_{4}\right)}, \delta=\frac{\left(3 k^{2}\left(A_{4}+\alpha_{0} B_{4}\right)^{2}-B_{4}^{2}\right)\left(k^{2}\left(A_{4}+\alpha_{0} B_{4}\right)^{2}+B_{4}^{2}\right)}{32 k^{2}\left(A_{4}+\alpha_{0} B_{4}\right)+B_{4}^{2}} \\
& A_{1}=\frac{A_{4} k^{2}\left(A_{4}+\alpha_{0} B_{4}\right)^{2}+B_{4}^{2}\left(A_{4}+2 \alpha_{0} B_{4}\right)}{k^{2}\left(A_{4}+\alpha_{0} B_{4}\right)^{2}+B_{4}^{2}}, A_{2}=0, A_{3}=0, A_{4}=A_{4} \\
& B_{1}=\frac{B_{4}\left(k^{2}\left(A_{4}+\alpha_{0} B_{4}\right)^{2}-B_{4}^{2}\right)}{k^{2}\left(A_{4}+\alpha_{0} B_{4}\right)^{2}+B_{4}^{2}}, B_{2}=0, B_{3}=0, B_{4}=B_{4}
\end{aligned}
$$


Substituting (3.46) into (3.10), we have :

$$
u(\xi)=\frac{\left(\alpha_{0} B_{4}+A_{4}\right)\left(B_{4}^{2}+k^{2}\left(\alpha_{0} B_{4}+A_{4}\right)^{2}\right)(\operatorname{sech}(\xi)+1)}{\left(k^{2} B_{4}\left(\alpha_{0} B_{4}+A_{4}\right)^{2}-B_{4}^{3}\right) \operatorname{sech}(\xi)+\left(k^{2} B_{4}\left(\alpha_{0} B_{4}+A_{4}\right)^{2}+B_{4}^{3}\right)}
$$

where $\quad \xi=x+\left(\frac{3 k^{2}\left(A_{4}+\alpha_{0} B_{4}\right)^{2}+B_{4}^{2}}{4 k B_{4}\left(A_{4}+\alpha_{0} B_{4}\right)}\right) t$

Substituting (3.47) into (3.8) and (3.6) yields

$$
\begin{aligned}
& h(\xi)=\sqrt{\frac{\left(\alpha_{0} B_{4}+A_{4}\right)\left(B_{4}^{2}+k^{2}\left(\alpha_{0} B_{4}+A_{4}\right)^{2}\right)(\operatorname{sech}(\xi)+1)}{\left(k^{2} B_{4}\left(\alpha_{0} B_{4}+A_{4}\right)^{2}-B_{4}^{3}\right) \operatorname{sech}(\xi)+\left(k^{2} B_{4}\left(\alpha_{0} B_{4}+A_{4}\right)^{2}+B_{4}^{3}\right)}} \\
& \psi(\xi)=\left(v+\left(\frac{3 k}{2}\right)\left(\frac{\left(\alpha_{0} B_{4}+A_{4}\right)\left(B_{4}^{2}+k^{2}\left(\alpha_{0} B_{4}+A_{4}\right)^{2}\right)(\operatorname{sech}(\xi)+1)}{\left(k^{2} B_{4}\left(\alpha_{0} B_{4}+A_{4}\right)^{2}-B_{4}^{3}\right) \operatorname{sech}(\xi)+\left(k^{2} B_{4}\left(\alpha_{0} B_{4}+A_{4}\right)^{2}+B_{4}^{3}\right)}\right)\right) d \xi \\
& =v \xi+\left(\frac{3 k E_{1}\left(E_{3}-E_{2}\right)}{E_{3} \sqrt{E_{2}^{2}-E_{3}^{2}}}\right) \arctan \left(\frac{\left(E_{2}-E_{3}\right) \tanh \left(\frac{\xi}{2}\right)}{E_{3} \sqrt{E_{2}^{2}-E_{3}^{2}}}\right)+\left(\frac{3 k E_{1}}{2 E_{3}}\right) \ln \left(\frac{\tanh \left(\frac{\xi}{2}\right)+1}{\tanh \left(\frac{\xi}{2}\right)-1}\right) \\
& E_{1}=\left(\alpha_{0} B_{4}+A_{4}\right)\left(B_{4}^{2}+k^{2}\left(\alpha_{0} B_{4}+A_{4}\right)^{2}\right) ; E_{2}=\left(k^{2} B_{4}\left(\alpha_{0} B_{4}+A_{4}\right)^{2}-B_{4}^{3}\right) \text {; } \\
& E_{3}=\left(k^{2} B_{4}\left(\alpha_{0} B_{4}+A_{4}\right)^{2}+B_{4}^{3}\right)
\end{aligned}
$$

Consequently,the exact solution of the derivative Schrödinger equation (3.1) with the help of Eq. (3.48) and Eq (3.2) are obtained in the following form:

$$
W(x, t)=\left[\begin{array}{l}
\sqrt{\left(\frac{\left(\alpha_{0} B_{4}+A_{4}\right)\left(B_{4}^{2}+k^{2}\left(\alpha_{0} B_{4}+A_{4}\right)^{2}\right)(\operatorname{sech}(\xi)+1)}{\left(k^{2} B_{4}\left(\alpha_{0} B_{4}+A_{4}\right)^{2}-B_{4}^{2}\right) \operatorname{sech}(\xi)+\left(k^{2} B_{4}\left(\alpha_{0} B_{4}+A_{4}\right)^{2}+B_{4}^{2}\right)}\right)} \times \\
\exp \left(\begin{array}{l}
\left(\begin{array}{l}
v \xi+\left(\frac{3 k E_{1}\left(E_{3}-E_{2}\right)}{E_{3} \sqrt{E_{2}^{2}-E_{3}^{2}}}\right) \\
i\left(\begin{array}{l}
\left(\frac{\left(E_{2}-E_{3}\right) \tanh \left(\frac{\xi}{2}\right)}{E_{3} \sqrt{E_{2}^{2}-E_{3}^{2}}}\right) \\
+\left(\frac{3 k E_{1}}{2 E_{3}}\right) \ln \left(\frac{\tanh \left(\frac{\xi}{2}\right)+1}{\tanh \left(\frac{\xi}{2}\right)-1}\right)-\left(\frac{\left(3 k^{2}\left(A_{4}+\alpha_{0} B_{4}\right)^{2}-B_{4}^{2}\right)\left(k^{2}\left(A_{4}+\alpha_{0} B_{4}\right)^{2}+B_{4}^{2}\right)}{32 k^{2}\left(A_{4}+\alpha_{0} B_{4}\right)+B_{4}^{2}}\right) t
\end{array}\right)
\end{array}\right)
\end{array}\right) \\
\xi=x+\left(\frac{3 k^{2}\left(A_{4}+\alpha_{0} B_{4}\right)^{2}+B_{4}^{2}}{4 k B_{4}\left(A_{4}+\alpha_{0} B_{4}\right)}\right) t
\end{array}\right)
$$

In particular setting $\quad \alpha_{0}=0, k=1, A_{4}=2, B_{4}=-1$ we find :

$$
\begin{aligned}
& W_{8}(x, t)=\left(\sqrt{-\frac{10(\operatorname{sech}(\xi)+1)}{3 \operatorname{sech}(\xi)+5}}\right) \times \exp \left(i\left(\begin{array}{l}
-3 \arctan \left(\frac{\tanh \left(\frac{\xi}{2}\right)}{2}\right)+\left(\frac{13}{8}\right) \xi \\
+3 \ln \left(\frac{\tanh \left(\frac{\xi}{2}\right)-1}{\tanh \left(\frac{\xi}{2}\right)+1}\right)-\left(\frac{55}{128}\right) t
\end{array}\right)\right) \\
& \xi=x-\frac{13}{8} t
\end{aligned}
$$

See Figure (3.8)

\section{Case 9:}

$$
\begin{aligned}
& \alpha_{0}=\alpha_{0}, v=v, \delta=-\frac{1}{2}\left(v^{2}+\frac{1}{4}\right) \\
& A_{1}=\frac{4 \alpha_{0} k^{2} v^{2}\left(A_{4}+\alpha_{0} B_{4}\right)-2 k v\left(A_{4}+2 \alpha_{0} B_{4}\right)+B_{4}}{k \sqrt{1+4 v^{2}}}, A_{2}=-\alpha_{0} k\left(A_{4}+\alpha_{0} B_{4}\right) \sqrt{1+4 v^{2}}, A_{3}=0, \\
& A_{4}=A_{4}, B_{1}=-\frac{2 v\left(2 v k\left(A_{4}+\alpha_{0} B_{4}\right)-B_{4}\right)}{\sqrt{1+4 v^{2}}}, B_{2}=k\left(A_{4}+\alpha_{0} B_{4}\right) \sqrt{1+4 v^{2}}, B_{3}=0, B_{4}=B_{4}
\end{aligned}
$$

Substituting (3.51) into (3.10), we have : 


$$
\begin{aligned}
& u(\xi) \\
& =\frac{\left(2 \alpha_{0} v B_{4}+2 v A_{4}-B_{4}\right) \operatorname{sech}(\xi)-\left(\alpha_{0} B_{4}+A_{4}\right) \sqrt{1+4 v^{2}}}{\left(2 \alpha_{0} v^{2} B_{4}-2 v B_{4}+4 v^{2} A_{4}\right) \operatorname{sech}(\xi)-\left(\alpha_{0} B_{4}+4 \alpha_{0} v^{2} B_{4}+A_{4}+4 v^{2} A_{4}\right) \cosh (\xi)-B_{4} \sqrt{1+4 v^{2}}}
\end{aligned}
$$

where $\quad \xi=x-v t$

Substituting (3.52) into (3.8) and (3.6) yields

$$
\begin{aligned}
& h(\xi)=\left(\sqrt{\frac{\left(2 \alpha_{0} v B_{4}+2 v A_{4}-B_{4}\right) \operatorname{sech}(\xi)-\left(\alpha_{0} B_{4}+A_{4}\right) \sqrt{1+4 v^{2}}}{\left(2 \alpha_{0} v^{2} B_{4}-2 v B_{4}+4 v^{2} A_{4}\right) \operatorname{sech}(\xi)-\left(\alpha_{0} B_{4}+4 \alpha_{0} v^{2} B_{4}+A_{4}+4 v^{2} A_{4}\right) \cosh (\xi)-B_{4} \sqrt{1+4 v^{2}}}}\right) \\
& \psi(\xi)=\left(\left(v+\left(\frac{3 k}{2}\right)\left(\frac{\left(2 \alpha_{0} v B_{4}+2 v A_{4}-B_{4}\right) \operatorname{sech}(\xi)-\left(\alpha_{0} B_{4}+A_{4}\right) \sqrt{1+4 v^{2}}}{\left(2 \alpha_{0} v^{2} B_{4}-2 v B_{4}+4 v^{2} A_{4}\right) \operatorname{sech}(\xi)-\left(\alpha_{0} B_{4}+4 \alpha_{0} v^{2} B_{4}+A_{4}+4 v^{2} A_{4}\right) \cosh (\xi)-B_{4} \sqrt{1+4 v^{2}}}\right)\right)\right) d \xi \\
& =\left(v+\frac{3}{2} k \alpha_{0}\right) \xi+3 \arctan \left(\left(-2 v+\sqrt{1+4 v^{2}}\right) \tanh \left(\frac{\xi}{2}\right)\right)+\frac{3}{2} k \alpha_{0} \ln \left(\frac{\tanh \left(\frac{\xi}{2}\right)-1}{\tanh \left(\frac{\xi}{2}\right)+1}\right)
\end{aligned}
$$

Consequently,the exact solution of the derivative Schrödinger equation (3.1) with the help of Eq. (3.53) and Eq. (3.2) are obtained in the following form:

$$
W(x, t)=\left(\begin{array}{l}
\left.\left(\sqrt{\frac{\left(2 \alpha_{0} v B_{4}+2 v A_{4}-B_{4}\right) \operatorname{sech}(\xi)-\left(\alpha_{0} B_{4}+A_{4}\right) \sqrt{1+4 v^{2}}}{\left(2 \alpha_{0} v^{2} B_{4}-2 v B_{4}+4 v^{2} A_{4}\right) \operatorname{sech}(\xi)-\left(\alpha_{0} B_{4}+4 \alpha_{0} v^{2} B_{4}+A_{4}+4 v^{2} A_{4} \cosh (\xi)-B_{4} \sqrt{1+4 v^{2}}\right.}}\right) \times\right) \\
\left.\exp \left(\begin{array}{l}
\left(v+\frac{3}{2} k \alpha_{0}\right) \xi+3 \arctan \left(\left(-2 v+\sqrt{1+4 v^{2}}\right) \tanh \left(\frac{\xi}{2}\right)\right) \\
i+\frac{3}{2} k \alpha_{0} \ln \left(\frac{\tanh \left(\frac{\xi}{2}\right)-1}{\tanh \left(\frac{\xi}{2}\right)+1}\right)+\frac{1}{2}\left(v^{2}+\frac{1}{4}\right) t
\end{array}\right)\right)
\end{array}\right)
$$

In particular setting $\alpha_{0}=1, v=1, k=2, A_{4}=1, B_{4}=1$ we find :

$$
W_{9}(x, t)=\left(\begin{array}{l}
\left(\frac{\sqrt{2}}{2}\right)\left(\sqrt{\frac{(7 \sqrt{5}) \operatorname{sech}(\xi)-(20)}{(14 \sqrt{5}) \operatorname{sech}(\xi)-(20 \sqrt{5}) \cosh (\xi)-5}}\right) \times \\
\left.\exp \left(\begin{array}{l}
\left.4 \xi+3 \arctan \left((-2+\sqrt{5}) \tanh \left(\frac{\xi}{2}\right)\right)\right) \\
+3 \ln \left(\frac{\tanh \left(\frac{\xi}{2}\right)-1}{\tanh \left(\frac{\xi}{2}\right)+1}\right)+\left(\frac{5}{8}\right) t
\end{array}\right)\right)
\end{array}\right)
$$

See Figure (3.9)

\section{Case 10:}

$$
\begin{aligned}
& \alpha_{0}=\alpha_{0}, v=-\frac{3 k^{2}\left(A_{3}+\alpha_{0} B_{3}\right)^{2}+9 B_{3}^{2}}{4 k B_{3}\left(A_{3}+\alpha_{0} B_{3}\right)}, \delta=\frac{3\left(k^{4}\left(A_{3}+\alpha_{0} B_{3}\right)^{4}+6 B_{3}^{2} k^{2}\left(A_{3}+\alpha_{0} B_{3}\right)^{2}-27 B_{3}^{4}\right),}{32 B_{3}^{2} k^{2}\left(A_{3}+\alpha_{0} B_{3}\right)^{2}}, \\
& A_{1}=-\frac{A_{3} k^{2}\left(A_{3}+\alpha_{0} B_{3}\right)^{2}+9 B_{3}^{2}\left(A_{3}+2 \alpha_{0} B_{3}\right)}{4 k^{2}\left(A_{3}+\alpha_{0} B_{3}\right)^{2}+36 B_{3}^{2}}, A_{2}=0, A_{3}=A_{3}, A_{4}=-\frac{3}{4} A_{3} \\
& B_{1}=-\frac{B_{3}\left(k^{2}\left(A_{3}+\alpha_{0} B_{3}\right)^{2}-9 B_{3}^{2}\right)}{4\left(k^{2}\left(A_{3}+\alpha_{0} B_{3}\right)^{2}+9 B_{3}^{2}\right)}, B_{2}=0, B_{3}=B_{3}, B_{4}=-\frac{3}{4} B_{3}
\end{aligned}
$$

Substituting (3.56) into (3.10), we have : 
The Generalized of $\cosh (\xi)$ Expansion Method And ItsApplication To Derivative Schrödinger..

$$
u(\xi)=\alpha_{0}+\frac{\left(-\frac{A_{3} k^{2}\left(A_{3}+\alpha_{0} B_{3}\right)^{2}+9 B_{3}^{2}\left(A_{3}+2 \alpha_{0} B_{3}\right)}{4 k^{2}\left(A_{3}+\alpha_{0} B_{3}\right)^{2}+36 B_{3}^{2}}\right) \operatorname{sech}(\xi)+A_{3} \cosh ^{2}(\xi)-\frac{3}{4} A_{3}}{\left(-\frac{B_{3}\left(k^{2}\left(A_{3}+\alpha_{0} B_{3}\right)^{2}-9 B_{3}^{2}\right)}{4\left(k^{2}\left(A_{3}+\alpha_{0} B_{3}\right)^{2}+9 B_{3}^{2}\right)}\right) \operatorname{sech}(\xi)+B_{3} \cosh ^{2}(\xi)-\frac{3}{4} B_{3}}
$$

where $\quad \xi=x+\left(\frac{3 k^{2}\left(A_{3}+\alpha_{0} B_{3}\right)^{2}+9 B_{3}^{2}}{4 k B_{3}\left(A_{3}+\alpha_{0} B_{3}\right)}\right) t$

Substituting (3.57) into (3.8) and (3.6) yields

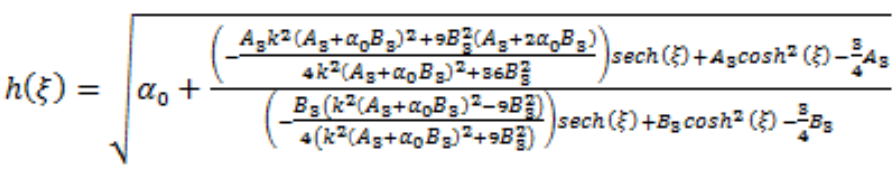

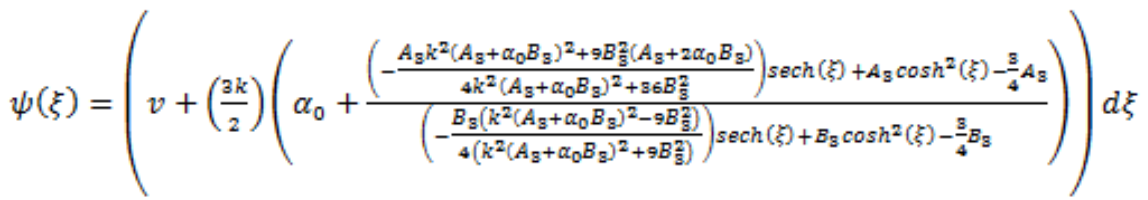

$$
\begin{aligned}
& =\left[\left(v+\frac{3 k}{2} \alpha_{0}\right) \xi+\left(\frac{3 k N_{2}}{2 N_{5}}\right) \ln \left(\frac{\tanh \left(\frac{5}{2}\right)+1}{\tanh \left(\frac{\xi}{2}\right)-1}\right)+\left(\frac{3 k}{N_{5}}\right) \sum_{\gamma} f(\gamma)\right] \\
& f(\gamma)=\left(\frac{\left(\left(N_{8} N_{5}+N_{2} N_{4}-N_{2} N_{6}-N_{1} N_{5}\right) \gamma^{4}+2\left(N_{1} N_{5}-N_{2} N_{4}\right) \gamma^{2}+\left(N_{2} N_{4}+N_{2} N_{6}-N_{8} N_{5}-N_{1} N_{5}\right)\right) \ln \left(\tanh \left(\frac{(5)}{2}\right)-\gamma\right)}{6\left(N_{4}-N_{5}-N_{6}\right) \gamma^{5}+4\left(N_{6}-3 N_{5}-3 N_{4}\right) \gamma^{8}+3\left(N_{6}-2 N_{5}+2 N_{4}\right) \gamma}\right) \\
& N_{1}=\left(-\frac{A_{g} k^{2}\left(A_{g}+\alpha_{0} B_{g}\right)^{2}+9 B_{s}^{2}\left(A_{g}+2 \alpha_{0} B_{g}\right)}{4 k^{2}\left(A_{g}+\alpha_{0} B_{8}\right)^{2}+36 B_{8}^{2}}\right), N_{2}=A_{3}, \\
& N_{3}=-\frac{3}{4} A_{3}, N_{4}=\left(-\frac{B_{8}\left(k^{2}\left(A_{8}+\alpha_{0} B_{8}\right)^{2}-9 B_{8}^{2}\right)}{4\left(k^{2}\left(A_{3}+\alpha_{0} B_{8}\right)^{2}+9 B_{8}^{2}\right)}\right), N_{5}=-\frac{3}{4} B_{3} \text {. } \\
& 0=\left(N_{4}-N_{5}-N_{6}\right) \gamma^{6}+\left(N_{6}-3 N_{5}-3 N_{4}\right) \gamma^{4}+\left(N_{6}+3 N_{4}-3 N_{5}\right) \gamma^{2}-\left(N_{4}+N_{5}+N_{6}\right)
\end{aligned}
$$

Consequently,the exact solution of the derivative Schrödinger equation (3.1) with the help of Eq. (3.58) and Eq. (3.2) are obtained in the following form:

$$
W(x, t)=\left[\begin{array}{l}
\left.\left(\sqrt{v+\left(\frac{3 k}{2}\right)\left(\alpha_{0}+\frac{\left(-\frac{A_{3} k^{2}\left(A_{3}+\alpha_{0} B_{3}\right)^{2}+9 B_{3}^{2}\left(A_{3}+2 \alpha_{0} B_{3}\right)}{4 k^{2}\left(A_{3}+\alpha_{0} B_{3}\right)^{2}+36 B_{3}^{2}}\right) \operatorname{sech}(\xi)+A_{3} \cosh ^{2}(\xi)-\frac{3}{4} A_{3}}{\left(-\frac{B_{3}\left(k^{2}\left(A_{3}+\alpha_{0} B_{3}\right)^{2}-9 B_{3}^{2}\right)}{4\left(k^{2}\left(A_{3}+\alpha_{0} B_{3}\right)^{2}+9 B_{3}^{2}\right)}\right) \operatorname{sech}(\xi)+B_{3} \cosh ^{2}(\xi)-\frac{3}{4} B_{3}}\right)}\right) \times\right] \\
\exp \left(i\left(\psi(\xi)-\left(\frac{3\left(k^{4}\left(A_{3}+\alpha_{0} B_{3}\right)^{4}+6 B_{3}^{2} k^{2}\left(A_{3}+\alpha_{0} B_{3}\right)^{2}-27 B_{3}^{4}\right)}{32 B_{3}^{2} k^{2}\left(A_{3}+\alpha_{0} B_{3}\right)^{2}}\right) t\right)\right.
\end{array}\right]
$$

$\xi=x+\left(\frac{3 k^{2}\left(A_{3}+\alpha_{0} B_{3}\right)^{2}+9 B_{3}^{2}}{4 k B_{3}\left(A_{3}+\alpha_{0} B_{3}\right)}\right) t$

In particular setting $\quad \alpha_{0}=1, k=1, A_{3}=4, B_{3}=2$ we find :

$$
W_{10}(x, t)=\left[\begin{array}{l}
\left(\sqrt{\left.1+\frac{\left(-\frac{3}{2}\right) \operatorname{sech}(\xi)+4 \cosh ^{2}(\xi)-3}{2 \cosh ^{2}(\xi)-\frac{3}{2}}\right)}\right) \times \\
\left.\exp \left(\begin{array}{l}
\left.-\frac{3}{2} \xi+3 \arctan \left(\tanh \left(\frac{\xi}{2}\right)\right)+3 \arctan \left(\frac{\tanh \left(\frac{\xi}{2}\right)}{\sqrt{3}-2}\right)\right) \\
-3 \arctan \left(\frac{\tanh \left(\frac{\xi}{2}\right)}{\sqrt{3}+2}\right)+3 \ln \left(\frac{\tanh \left(\frac{\xi}{2}\right)+1}{\tanh \left(\frac{\xi}{2}\right)-1}\right)-\left(\frac{9}{8}\right) t
\end{array}\right)\right)
\end{array}\right]
$$

See Figure (3. 10)

$$
\xi=x+3 t
$$




\section{Table Graphics}

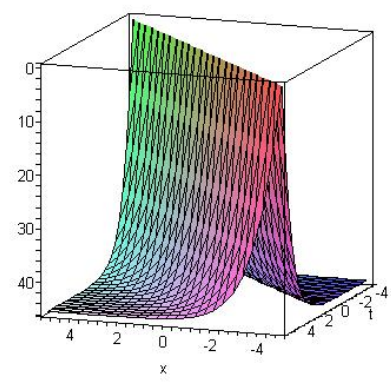

Figure (3.1)

The 3D plot of $\left|\mathrm{W}_{1}(\xi)\right|$

$\xi=x+t$
$-5 \leq x \leq 5$
$-5 \leq t \leq 5$

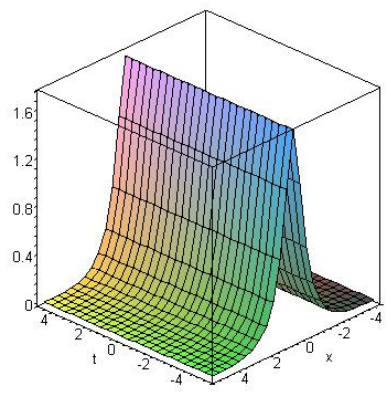

Figure (3.4)

The 3D plot of $\left|\mathrm{W}_{4}(\xi)\right|$

$$
\begin{gathered}
\xi=\mathrm{x} \\
-5 \leq \mathrm{x} \leq 5 \\
-5 \leq \mathrm{t} \leq 5
\end{gathered}
$$

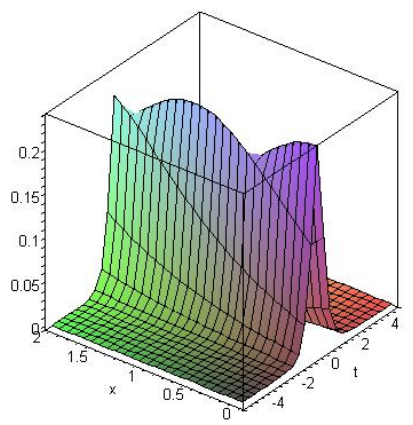

Figure (3.7)

The 3D plot of $\left|\mathrm{W}_{7}(\xi)\right|$

$$
\begin{gathered}
\xi=\mathrm{x}+\left(\frac{35}{12}\right) \mathrm{t} \\
-5 \leq \mathrm{x} \leq 5 \\
-5 \leq \mathrm{t} \leq 5
\end{gathered}
$$

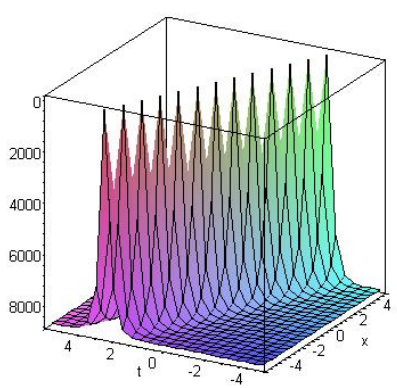

Figure (3.2)

The 3D plot of $\left|\mathrm{W}_{2}(\xi)\right|$

$$
\begin{gathered}
\xi=\mathrm{x}+2 \mathrm{t} \\
-5 \leq \mathrm{x} \leq 5 \\
-5 \leq \mathrm{t} \leq 5
\end{gathered}
$$

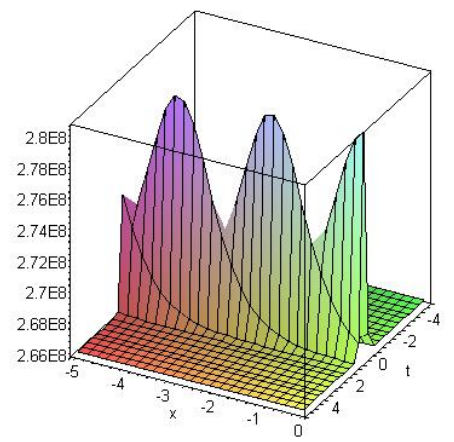

Figure (3.5)

The 3D plot of $\left|\mathrm{W}_{5}(\xi)\right|$

$$
\begin{gathered}
\xi=\mathrm{x}+\left(\frac{14}{3}\right) \mathrm{t} \\
0 \leq \mathrm{x} \leq 5 \\
-5 \leq \mathrm{t} \leq 5
\end{gathered}
$$

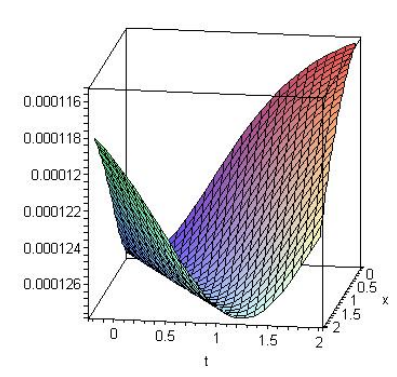

Figure (3.8)

The 3D plot of $\left|\mathrm{W}_{8}(\xi)\right|$

$$
\begin{gathered}
\xi=\mathrm{x}-\left(\frac{13}{8}\right) \mathrm{t} \\
0 \leq \mathrm{x} \leq 2 \\
0 \leq \mathrm{t} \leq 2
\end{gathered}
$$

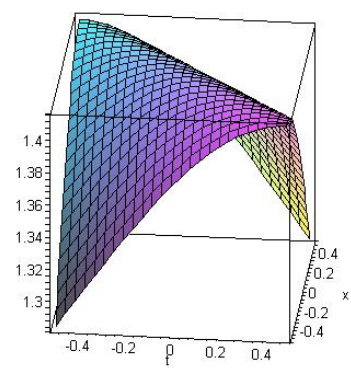

Figure (3.3)

The 3D plot of $\left|\mathrm{W}_{3}(\xi)\right|$

$$
\xi=\mathrm{x}+\mathrm{t}
$$

$-0.5 \leq \mathrm{x} \leq 0.5$

$-0.5 \leq \mathrm{t} \leq 0.5$

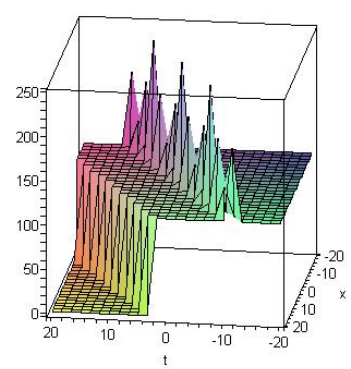

Figure (3.6)

The 3D plot of $\left|\mathrm{W}_{6}(\xi)\right|$

$$
\begin{aligned}
& \xi=\mathrm{x}+\left(\frac{7}{4}\right) \mathrm{t} \\
& -20 \leq \mathrm{x} \leq 20 \\
& -20 \leq \mathrm{t} \leq 20
\end{aligned}
$$

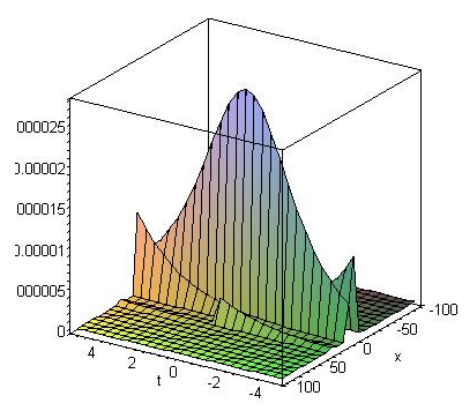

Figure (3.9)

The 3D plot of $\left|\mathrm{W}_{9}(\xi)\right|$

$$
\begin{gathered}
\xi=\mathrm{x}-\mathrm{t} \\
-5 \leq \mathrm{x} \leq 5 \\
-100 \leq \mathrm{t} \leq 100
\end{gathered}
$$




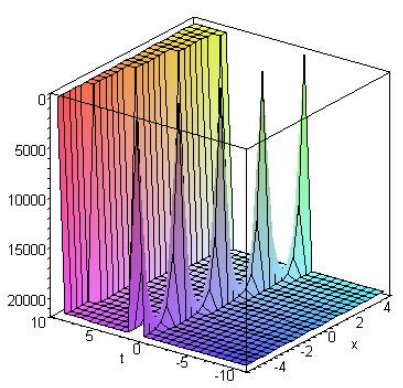

Figure(3.10)

The 3D plot of $\left|\mathrm{W}_{10}(\xi)\right|$

$\xi=\mathrm{x}+3 \mathrm{t}$

$-5 \leq \mathrm{x} \leq 5$

$-10 \leq \mathrm{t} \leq 10$

\section{Conclusion}

In this article, we propose new technique called The generalized of $\cosh (\xi)$ expansion method. this method has been applied to find the exact traveling solutions of the Derivative Schrödinger Equation. these solutions have rich local structures, It may be important to explain some physical phenomena . This work shows that, the generalized of $\cosh (\xi)$ expansion method is direct, effective and can be used for many other NLPDEs in mathematical physics.

\section{References}

[1]. M. Wang, X. Li, and J.Zhang (2008).The $\left(\frac{G}{G}\right)$ expansion mathod and travelling wave solutions of nonlinear evolution equations in mathematical physics, Physics Letters A, Vol. 372, no. 4,:417-423.

[2]. Mohammad Ali Bashir, Alaaedin Amin Moussa (2014). New Approach of $\left(\frac{G}{G}\right)$ Expansion Method. Applications to KdV Equation, Journal of Mathematics Research, Vol. 6, No. 1: 24-31.

[3]. L.X.Li, E.q.Li and M. wang (2010).The $\left(\frac{G^{\prime}}{G}, \frac{1}{G}\right)$ expansion method and its application to travelling wave solutions of the Zakharov equation, Applied Mathematics B, Vol. 25, No. 4:454-462.

[4]. M Ali Akbar and Norhashidah Hj Mohd Ali (2014).Solitary wave solutions of the fourth order Boussinesq equation through $\exp (-\phi(\xi))$ the expansion method, Springer plus ,3:344.

[5]. K. Khan and M.A. Akbar (2013). Application of $\exp (-\phi(\xi))$ expansion method to find the exact solutions of modified Benjamin-Bona-Mahony equation, World Applied Sciences Journal, 24(10):1373-1377.

[6]. Mohammad Ali Bashir, Alaaedin Amin Moussa, Lama Abdulaziz Alhakim (2015). The new generalized of exp $(-\phi(\xi))$ expansion method and its application to some complex nonlinear partial differential equation, Journal of advanced in Mathematics, Vol.9, No. 8:2905-2926.

[7]. Khalil Hassan Yahya, Zelal Amin Moussa, (2015). new approach of generalized exp $(-\phi(\xi))$ expansion method and its application to some nonlinear partial differential equation, Journal of Mathematics research, Vol.7, No.3:106-121.

[8]. M.Inc and M, Ergut, (2005).Periodic Wave Solutions for the Generalized Shallow Water wave Equation by the Improved Jacobi Elliptic Function Method, Appl. Math. E-Notes.,5:89-98.

[9]. C. A. Gomez, A. H. Salas, and B. A. Frias(2010).Exact solutions to KdV6 equation by using a new approach of the projective Riccati equation method,Mathematical Problems in Engineering, Article ID 797084, 2010, doi: 10.1155/2010/797084, (10 pages).

[10]. S. Guo, L. Mei, Y. Zhou and C. Li, (2011). The extended Riccati equation mapping method for variable-coefficient diffusion-reaction and $m K d V$ equation, Applied Mathematics and Computation, vol. 217: 6264--6272.

[11]. E. Yusufoglu and A, Bekir, (2006).Solitons and Periodic Solutions of Coupled Nonlinear Evolution Equations by Using Sine-Cosine Method, Internat J. Comput. Math., 83(12):915-924.

[12]. Mohammad Ali Bashir, Lama Abdulaziz Alhakim (2013).New F Expansion method and its applications to Modified KdV equation, Journal of Mathematics Research, Vol. 5, No. 4 38-94.

[13]. M. J.Ablowitz, G.Biondini and S. De Lillo (1997).On the Well-posedness of the Eckhaus Equation", physics Letters A 230., 319-323.

[14]. M. Mirzazadeh, S. Khaleghizadeh (2013). Modification of truncated expansion method to some complex nonlinear partial differntial equations, Acta Universitatis Apulensis, No 33:109-116.

[15]. S.T. Mohyud-Din and M. A. Noor (2008).Solving Schrödinger Equations by Modified Variational Iteration Method", World Appl. Science J., 5(3):352-357.

[16]. Mohammad Ali Bashir, Alaaedin Amin Moussa (2014).The coth c $_{a}(\xi)$ Expansion Method and its Application to the Davey-Stewartson Equation, Applied Mathematical Sciences, Vol. 8, no. 78,: 3851-3868. 